\title{
PREVENTION OF SUMMER OVERHEATING OF BUILDINGS WITH ADDED CONVEX WINDOWS
}

\author{
A. KEREKES ${ }^{1, \mathrm{a}}, C S . R A ́ C Z^{2, \mathrm{~b}}, A . C S . D O B O S^{2, \mathrm{c}}$ \\ ${ }^{1}$ Department of Building Services and Building Engineering, Faculty of Engineering, University of Debrecen, Debrecen, Hungary \\ ${ }^{2}$ AKIT DTTI Agrometeorological and Agro-ecological Monitoring Centre, University of Debrecen, Debrecen, Hungary \\ aE-mail: kerekesa@eng.unideb.hu \\ bE-mail: raczcs@agr.unideb.hu \\ cE-mail: dobosa@agr.unideb.hu
}

\begin{abstract}
When renovating an existing building, keeping the original window and installing added convex window is beneficial for the reduction of heating energy demand. This solution is free of the disposal and/or recycling of demolition waste. In the summer season as far as the transparent structures of the building are concerned, in general, heat protection has to be used. In the case of the added convex window, it can be provided by the external air curtain mode; the movable shading device is in the buffer zone. This is a well-protected place for it, so that it can be as simple and cheap as an inner shading device. In this article examinations of the efficiency of the external air curtain mode for the heat protection is presented. The summer overheating has been analysed in two experimental huts. It has been proven that simple shading device in added convex window tempers the summer overheating as efficiently as the combination of usual window and external shading device (which is exposed to heavy weather effects and is difficult to maintain).
\end{abstract}

Keywords: added convex window, summer overheating, shading efficiency, experimental huts

\section{Introduction}

When renovating an existing building, it is beneficial to keep the original window and to use the added convex window for the reduction of heating energy demand (Fig. 1), which solution is free of the disposal and recycling of demolition waste. Installation of the added convex window is not or only moderately disturbing the tenants. By using this structure it is possible to channel air flow through the added convex window. It is also beneficial for reducing the energy need for heating [1-3], but in this article the topic is the summer heat protection. In the summer season, as for the transparent structures of the building in general, heat protection has to be used $[4,5]$. In the case of traditional windows an external shading device can be an efficient solution. In the case of the added convex window it can be provided by the external air curtain mode
(Fig. 2). In this case the mobile shading device is in a protected place, it can be as simple and cheap as inner shading device. The natural air movement provides the cooling of the mobile shading device by the opening of the added convex window on the upper and lower part.

In this article we would like to compare the efficiency of these solutions. Concerning the efficiency of the heat protection we could talk about energy saving, but in dwellings the aim is to provide the acceptable thermal comfort without mechanical cooling. The efficiency of the heat protection was therefore evaluated by the inner temperature experienced inside the building.

\section{Methods}

The evaluation of the heat protection was based on measurements. The principle of choosing measure-

This is an open-access article distributed under the terms of the Creative Commons Attribution-NonCommercial 4.0 International License (https://creativecommons.org/licenses/by-nc/4.0/), which permits unrestricted use, distribution, and reproduction in any medium for non-commercial purposes, provided the original author and source are credited, a link to the CC License is provided, and changes - if any - are indicated. 


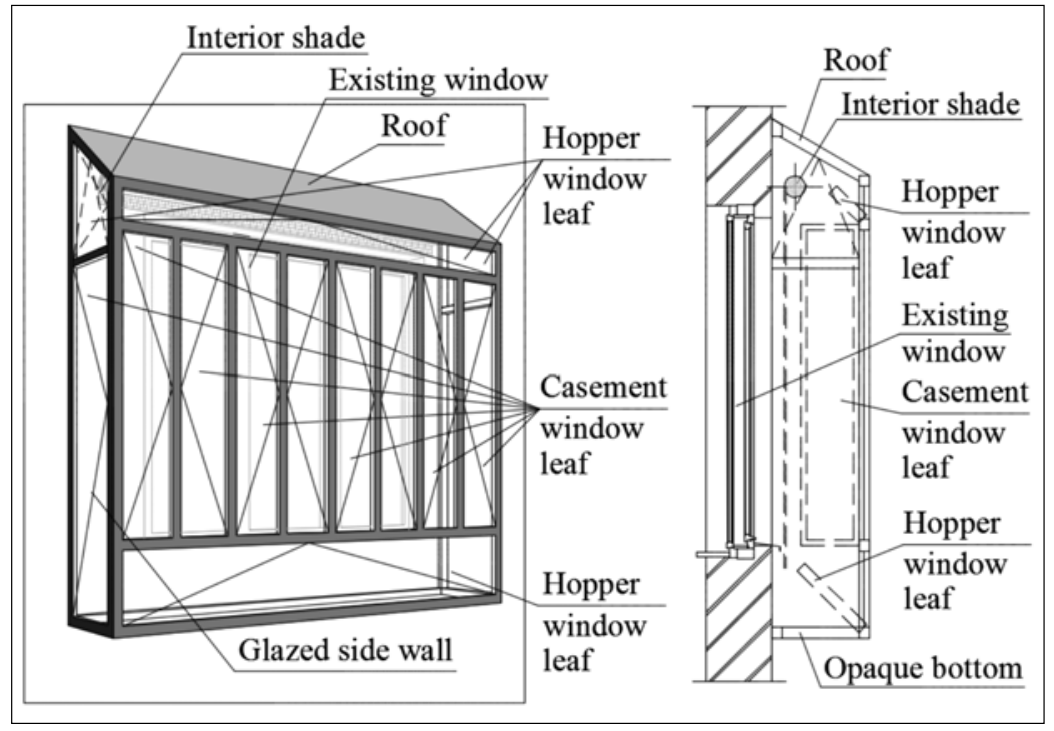

Fig. 1. Sketch of a possible form of the added convex window

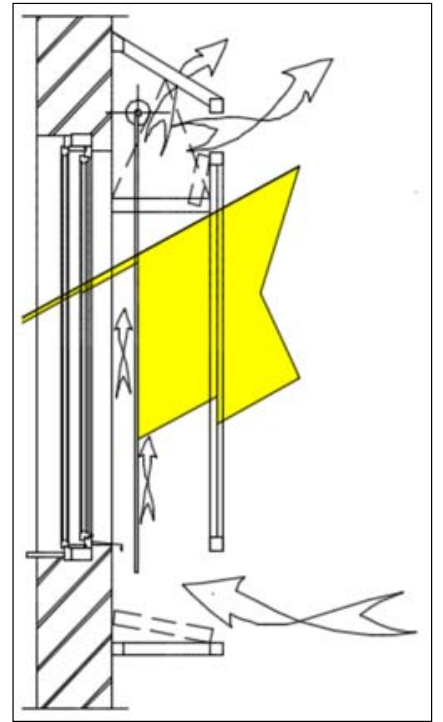

Fig. 2. Sketch of added convex window operating in external air curtain mode ments method was that the energetic impact of the added convex window had to be shown clearly and numerically and it had to be appropriate for the evaluation of the risk of summer overheating and summer heat protection. Measurements were carried out in two identical experimental huts with only one difference: one of them had an added convex window.

The following parameters were exactly the same: the sizes, the used material and their technical traits, the technology of the constructions, the mode of operation and use, the mode of ventilation and its amount, the placement method and the geographic and meteorological situation of the building.
Based on the close relationship of the parameters and circumstances of the measurements, the difference between the results of the measurements, carried out simultaneously in the two huts, can only be caused by the added convex window and the position of the shading device. Thus the impact of added convex window for the summer overheating can be shown trustworthily by this method.

In order to meet these expectations two identical experimental huts have been placed in the same place with the same orientation. During the summer season the inner temperature was registered in different cases of shadings. The orientations were changeable by the

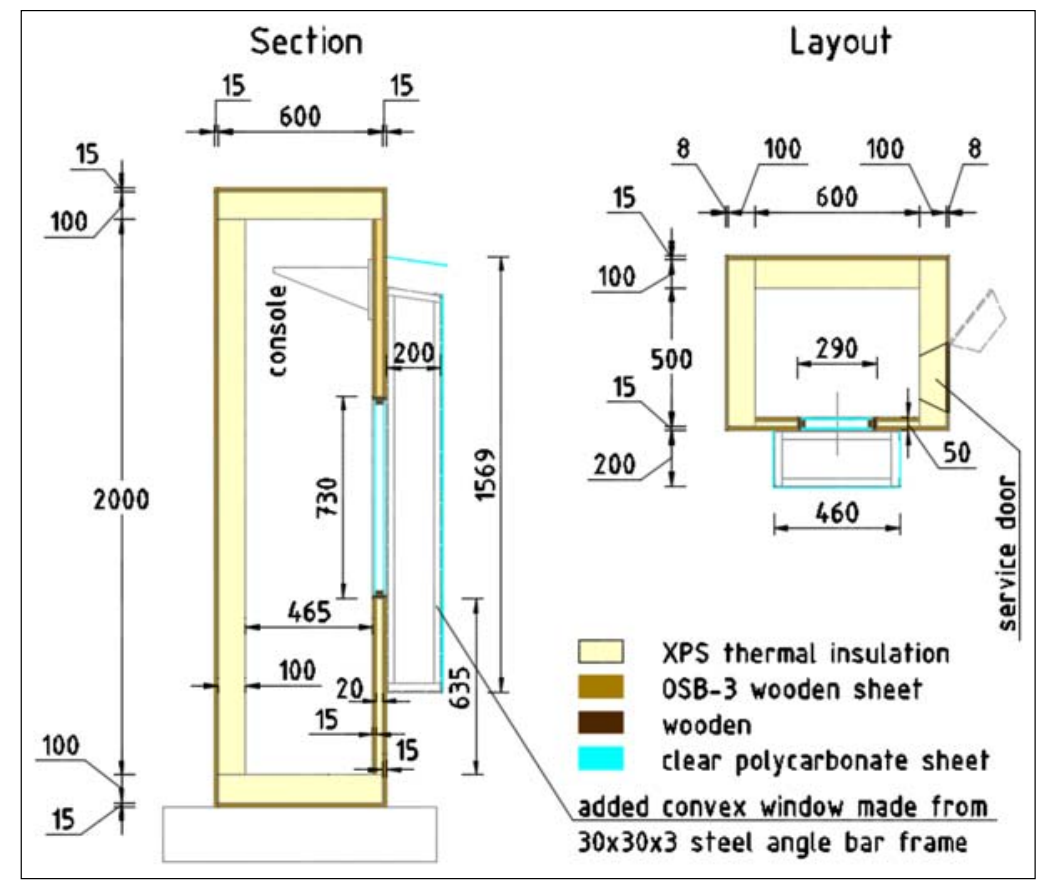

Fig. 3. Layout and section of experimental hut with added convex window 
rotation of the experimental huts. The layout and the section of the huts can be seen in Fig. 3. The size of the huts were chosen according to the followings: the rotations of them had to be done manually, so we tried to minimise the sizes, on the other hand, the height of the huts could not be less than $2 \mathrm{~m}$ since the validation limits of the modelling had to be considered. The wall structure is made of XPS thermal insulation, which is connected to the OSB-3 boards covering by using EPS foam, which guaranteed the continuity of the thermal insulation at the joints. The huts were constructed in the summer (Fig. 4). The external facades were painted by 3 layers of painting. For sup-

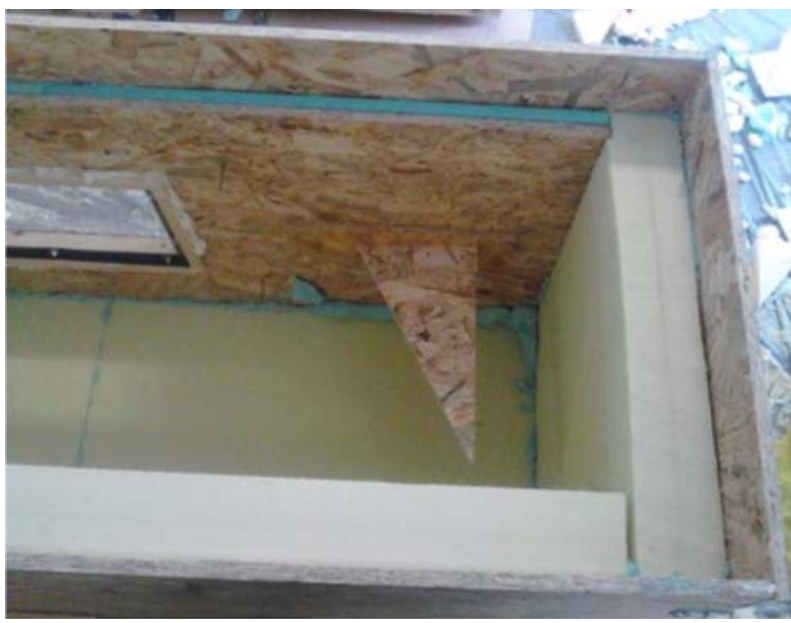

Fig. 4. Experimentel hut during its construction porting the measuring and the data logger a console was placed. The transparent structures were made of polycarbonate instead of glass because of the effects during the delivery and the mobilisation. The solar heat gain coefficient (SHGC) of the two materials do not differ significantly. The window total thermal performance indices were calculated by Berkeley Lab WINDOW v7.4.8.0 software. Due to the results of the simulations the $U$-value of the wall equals the $U$ value of a B-30 brick wall. The $U$ value of the polycarbonate window is equal to the $U$ value of a wooden-frame double-glazed window. The other three walls, the floor and the roof were made of structure with lower $U$ value than the $U$ value of the B- 30 brick wall, because the aim was that the measurements show the impact of the added convex window. The huts have service door for handling the measuring devices. The huts were placed on wooden structures. The stabilisation of them were provided by steel chains, in four directions. (Fig. 6) The temperature data were collected by KIMO KTH100 and KTH-300 data loggers.

The experimental huts have been installed at the Agrometeorological Observatory of the AKIT DTTI Agrometeorological and Agro-ecological Monitoring Centre, University of Debrecen (Fig. 7), where continuous data series are available in high resolution. It is located in Debrecen-Kismacs, the outskirts of Debrecen, $2 \mathrm{~km}$ North-west of the city at Lat: $47.577^{\circ}$, Lon: $21.582^{\circ}, 125 \mathrm{~m} \mathrm{ASL}$. It is a slightly undulating, plain, open area, relatively higher than the surrounding ag-

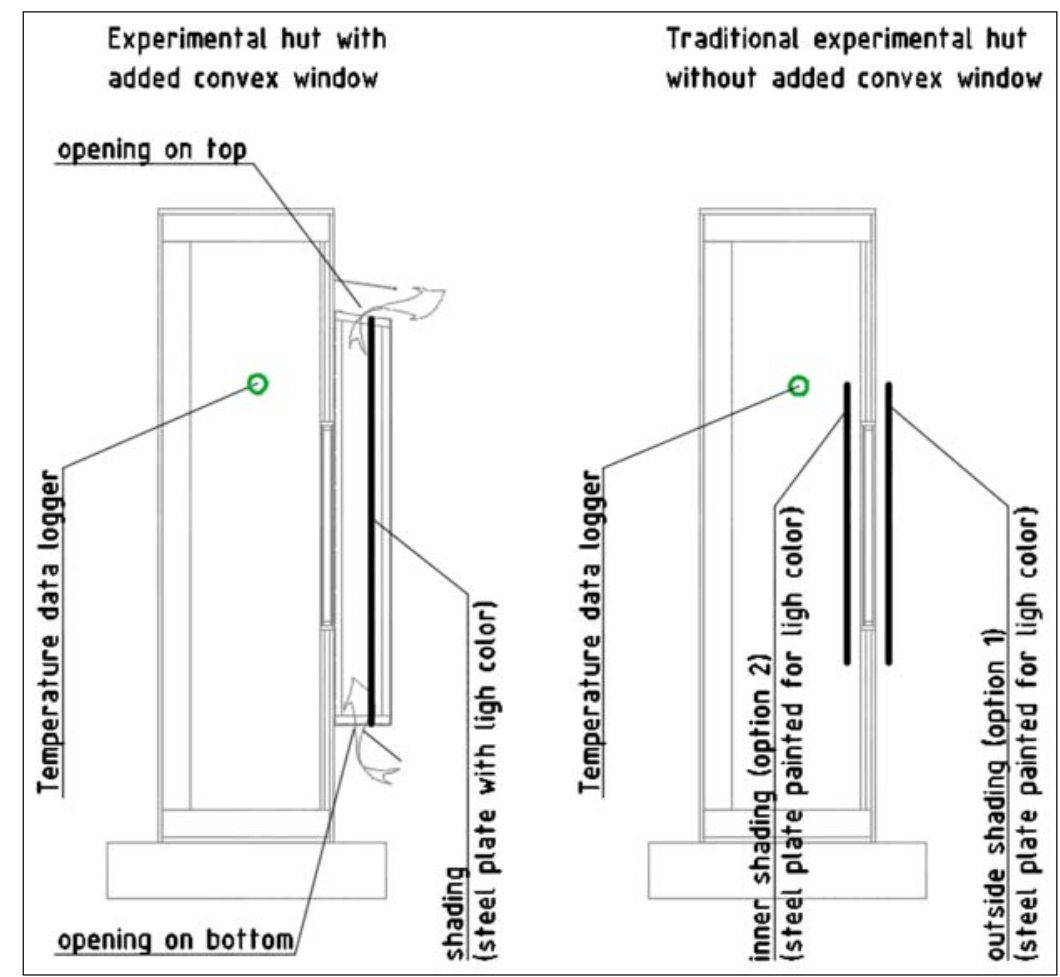

Fig. 5. Experimental huts in summer mode (location of sensors and shading devices) 
Table 1. Main characteristics of the measured parameters at the Observatory

\begin{tabular}{lclccc}
\hline Parameter & Height $(\mathrm{m})$ & Type of instrument & Increment $(\mathrm{min})$ & Accuracy & Resolution \\
\hline Dry-bulb air temperature & $1-2$ & Pt100-1/10 & 10 & $\pm 0.1^{\circ} \mathrm{C}$ & $0.1^{\circ} \mathrm{C}$ \\
Relative humidity & $1-2$ & Vaisala HMP-155 & 10 & $\pm 1-1.7 \%$ & $0.1 \%$ \\
Wind speed \& direction & $1-2-4-10$ & Vaisala WAA-151 & 10 & $\pm 0.5 \mathrm{~m} \mathrm{~s}^{-1}$ & $0.1 \mathrm{~m} \mathrm{~s}^{-1}$ \\
Global radiation & 2 & K\&Z CMP-11 & 10 & $\pm 1 \%$ & $0.1 \mathrm{~W} \mathrm{~m}^{-2}$ \\
\hline
\end{tabular}

ricultural land. The natural vegetation is low, mixed grass association cropped to $5 \mathrm{~cm}$ surrounded by cultivated fields of maize, wheat, barley and sunflower. The Observatory also serves as a qualified weather station of the Hungarian Meteorological Service with where

$I_{b n}$ the hourly direct normal radiation, $\mathrm{Wh} / \mathrm{m}^{2}$

$I$ the hourly global radiation, $\mathrm{Wh} / \mathrm{m}^{2}$

$I_{d}$ the hourly diffuse radiation, $\mathrm{Wh} / \mathrm{m}^{2}$

$\theta_{z}$ the zenit angle of the sun.

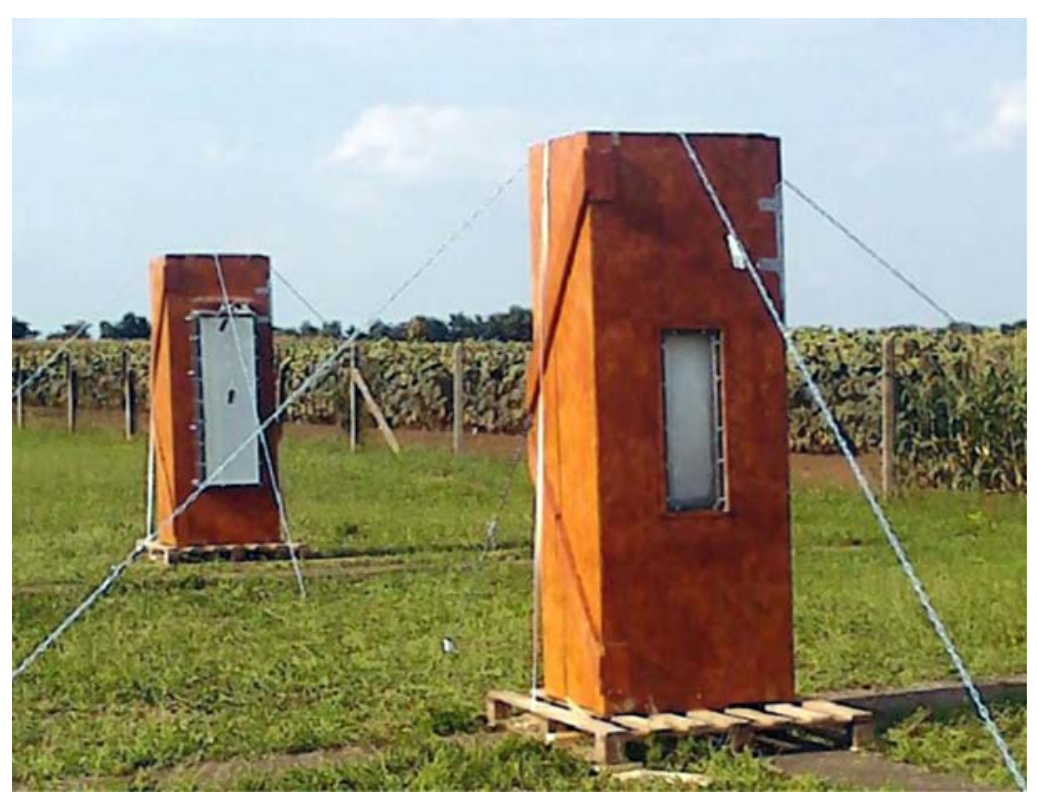

Fig. 6. Experimental huts in summer mode (South orientation, the traditional hut is with inner shading device)

its wide range of specific measurements. The establishment of the measurement program dates back to 1964, it has been significantly extended in 2008 and the instrumentation has also been renewed that year. Characteristics of the used meteorological data and measurements are listed in Table 1.

The measurements of the one part of the meteorological parameters were carried out in the place of the experimental huts - Agrometeorological Observatory - other data were available from www.ogimet.com internet site, which is a professional meteorological information site, data can be downloaded if demanded. These data are from the Debrecen Meteorological Station number 12882, which is situated $12 \mathrm{~km}$ from the Agrometeorological Observatory. The data are from synop reports.

After all these data have been gathered the following calculations are needed.

The hourly direct normal radiation [6]:

$$
I_{b n}=\left(I-I_{d}\right) \cos \theta_{z}
$$

The hourly radiation data is for the whole hours for the starting point of the hour (ex. 10:00 means 9:00 $-9: 59$ period), so midpoint of the hours must be used for the angles and radiation values).

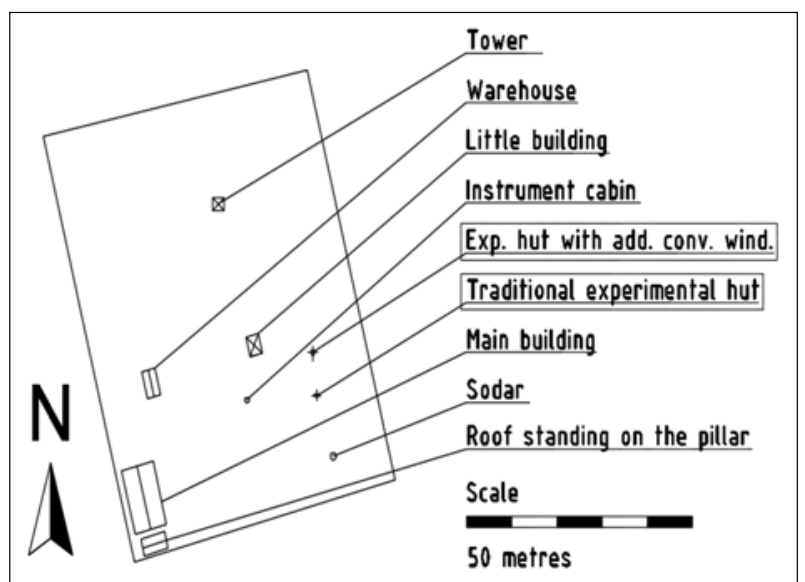

Fig. 7. Placement of the experimental huts and the surrounding buildings and objects 
Cosinus of the zenit angle of the Sun:

$$
\cos \theta_{z}=\cos \varphi \cos \delta \cos \omega+\sin \varphi \sin \delta,
$$

where

$\varphi$ the latitude, ${ }^{\circ}$

$\delta$ the declination, ${ }^{\circ}$

$\omega$ the hour angle.

The declination $[7,8]$ :

$$
\begin{gathered}
\delta=(180 / \pi)(0.006918-0.399912 \cos B \\
+0.070257 \sin B-0.006758 \cos 2 B \\
+0.000907 \sin 2 B-0.002697 \cos 3 B \\
+0.00148 \sin 3 B), \\
B=(n-1)(360 / 365),
\end{gathered}
$$

where

$n \quad$ the $n$th day of the year (1st January: $n=1)$.

The hour angle $[7,8]$ :

$$
\omega=15(\text { solar time }-12) .
$$

The solar time $[7,8]$ :

solar time $=$ standard time $+4\left(L_{\mathrm{st}}-L_{\mathrm{loc}}\right)+E,(6)$

where

$L_{\text {loc }}$ the longitude of the location (longitudes are in degrees West), ${ }^{\circ}$

$L_{\text {st }}$ the standard meridian of the local time zone, ${ }^{\circ}$

$E$ the equation of time, min. CET.

The standard time is in Central European Time,

$$
\begin{aligned}
E= & 229.2(0.000075+0.001868 \cos B \\
& -0.032077 \sin B-0.014615 \cos B \\
& -0.04089 \sin 2 B) .
\end{aligned}
$$

The hourly diffuse radiation (the calculated diffuse part of the measured global radiation) [9]:

$$
I_{d}=\left(I_{d} / I\right) I,
$$

where

$\left(I_{d} / I\right)$ the diffuse fraction:

$$
\frac{I_{d}}{I}=\left\{\begin{array}{ccc}
1.0-0.09 k_{T}, & \text { if } & k_{T} \leq 0.22 \\
0.9511-0.1604 k_{T} & & \\
+4.388 k_{T}^{2}-16.638 k_{T}^{3} & \text { if } & 0.22<k_{T} \leq 0.8 \\
+12.336 k_{T}^{4}, & & \\
0.165, & \text { if } & k_{T}>0.8
\end{array}\right.
$$

The hourly clearness index:

$$
k_{T}=I / I_{0},
$$

where

$k_{T}$ the hourly clearness index,

$I_{0}$ the hourly extraterrestrial radiation, $\mathrm{Wh} / \mathrm{m}^{2}$.

The hourly extraterrestrial normal radiation $[7,8]$ :

$$
\begin{aligned}
I_{0 n}= & G_{\mathrm{SC}}(1.000110+0.034221 \cos B \\
& +0.001280 \sin B+0.000719 \cos 2 B \\
& +0.000077 \sin 2 B),
\end{aligned}
$$

where

$G_{\mathrm{SC}}$ the solar constant, $\mathrm{W} / \mathrm{m}^{2}$,

$I_{0 n}$ the hourly extraterrestrial normal radiation, $\mathrm{Wh} / \mathrm{m}^{2}$.

The hourly extraterrestrial horizontal radiation:

$$
I_{0}=I_{0 n} \cos \theta_{z} .
$$

If we are in the hour of the sunrise, the calculation of the extraterrestrial horizontal radiation must be done for the period starting with sunrise till the next whole hour with the following equation (in the case of the sunset the calculation has to be done similarly, the period starting with the whole hour before the sunset till the sunset) $[6,9]$ :

$$
\begin{aligned}
I_{0}= & (12 / \pi) I_{0 n}\left[\cos \varphi \cos \delta\left(\sin \omega_{2}-\sin \omega_{1}\right)\right. \\
& \left.+(\pi / 180)\left(\omega_{2}-\omega_{1}\right) \sin \varphi \sin \delta\right] .
\end{aligned}
$$

The hour angel of the sun rise and sun set:

$$
\omega_{s}=\arccos (-\tan \varphi \tan \delta) .
$$

If we did not calculate in the above-mentioned way for the hour of sunrise or sunset, we would receive too low extraterrestrial horizontal radiation value, which would result in big hourly clearness index, and therefore small hourly diffuse radiation ratio and big direct radiation ratio. The big direct radiation value would lead to unrealistic big direct normal radiation value, because of the big zenit angle of the sun.

\section{Results}

During the measurements the inner temperature of the huts have been registered by data loggers for the following cases:

Experimental hut with added convex window:

- shading device in the buffer zone, on the upper and lower part of the added convex window opening for natural ventilations, the area of the opening equals the horizontal area of the added convex window, 
In traditional experimental hut:

- inner shading device,

- outer shading in the front of the window (Fig. 5).

Figure 8 shows the radiation data for the given days (calculated and measured data). (The measured data are from Agrometeorological Observatory of the AKIT DTTI Agrometeorological and Agro-ecological Monitoring Centre, University of Debrecen, Hungary.)

The temperature measurements were carried out in the southern and western directions. Results of a few days are shown in Fig. 9.

\section{Discussion}

Improving the thermal insulation of the building can lead to increasing the risk of summer overheating. The indirect passive solar heating systems with greenhouse effect need protection against summer overheating. One of the energetic effects of the added convex windows is the decreasing of the heat transfer coefficient, the other is the significant greenhouse effect. Based on all these it is necessary to reduce the risk of the summer overheating and we need to make sure that the suggested method is efficient.

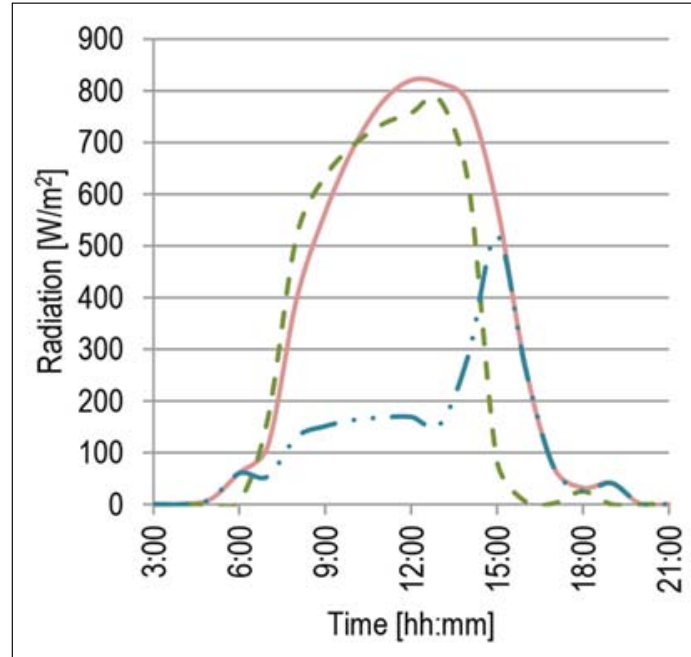

a) 6th August 2016

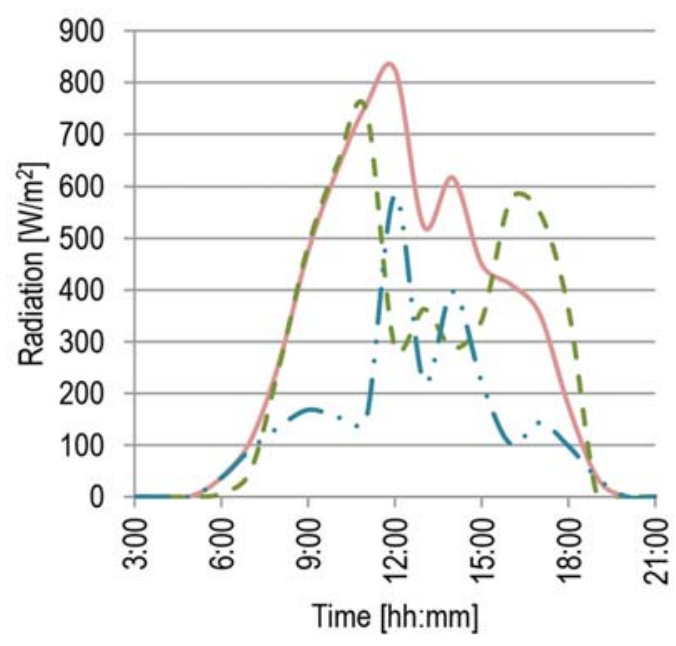

Legend:

c) 13th August 2016

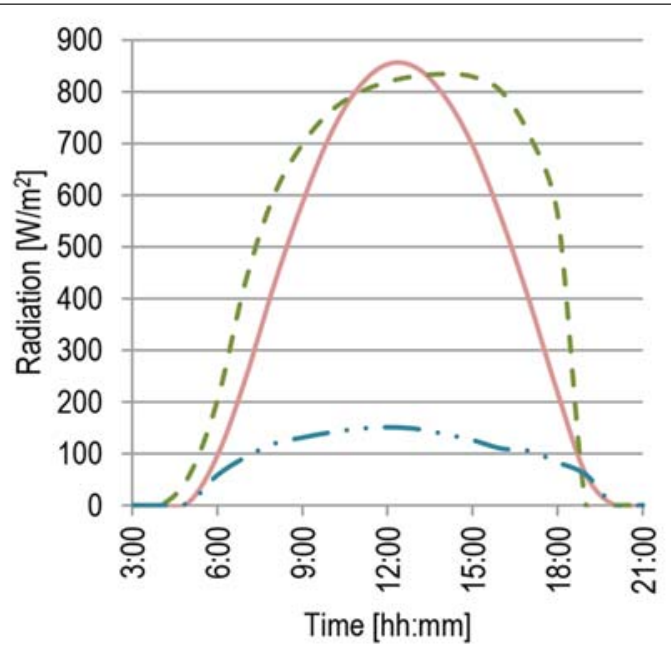

b) 7 th August 2016

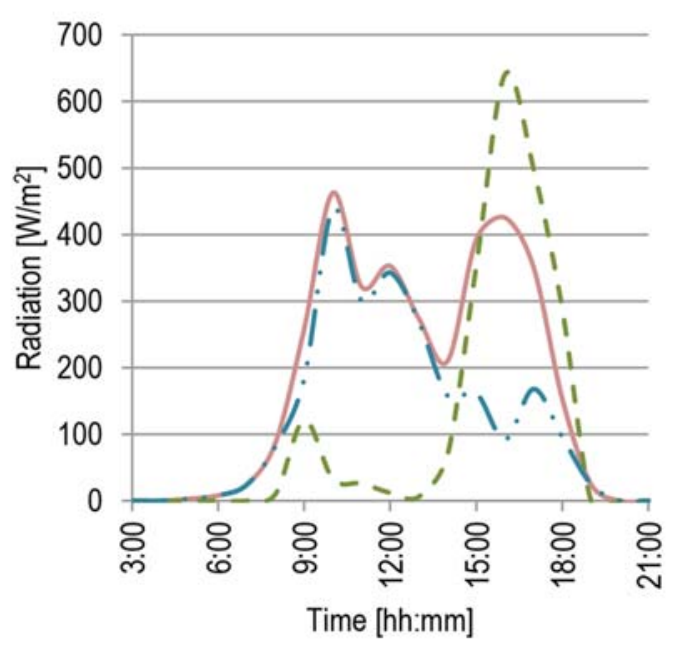

d) 17 th August 2016

Global Horizontal Radiation (measured)

- $\cdots-\quad$ Diffuse Horizontal Radiation (calculated)

- - - - Direct Normal Radiation (calculated)

Fig. 8. Radiation data (time: Central European Time, CET) 
The results of the measured data for the two experimental huts concerning overheating show that the inner shading placed in the buffer zone of the added convex window is significantly more beneficial compared to the other hut with the inner shading device.

Figure 8 shows that in the case of southern orientation the overheating of the experimental hut with added convex window is lower by $9.18^{\circ} \mathrm{C}$ than in the traditional huts. And in the case of western orientation it is lower by $11^{\circ} \mathrm{C}$.
The inner shading device placed in the buffer zone is equally efficient, even a bit more beneficial than the outside shading device used on the traditional hut. The overheating of the huts were practically the same.

\section{Conclusions}

These measured data have proved that the simple shading device in added convex window can temper the summer overheating as well as the external shading device (exposed to heavy weather effects and dif-

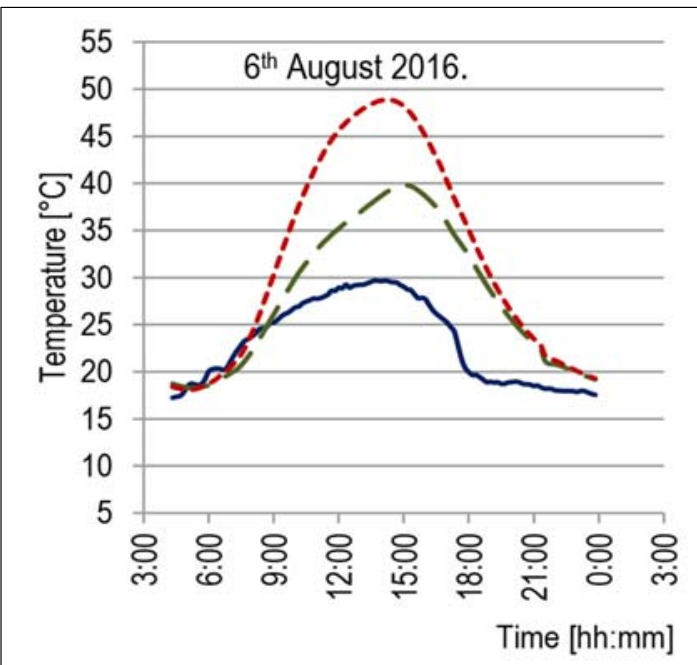

a) South orientation

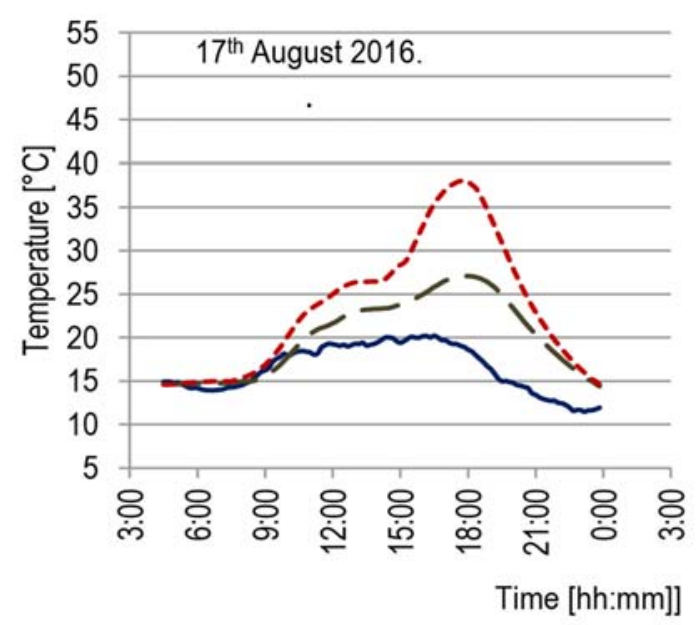

c) West orientation

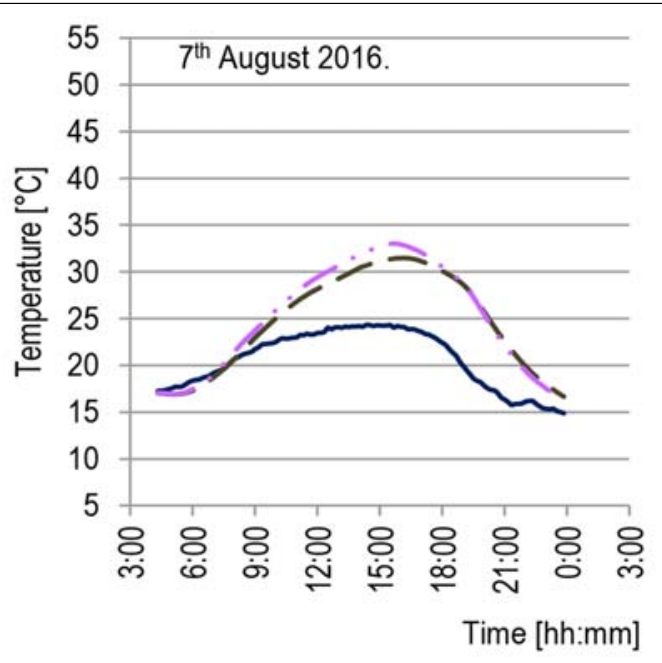

b) South orientation

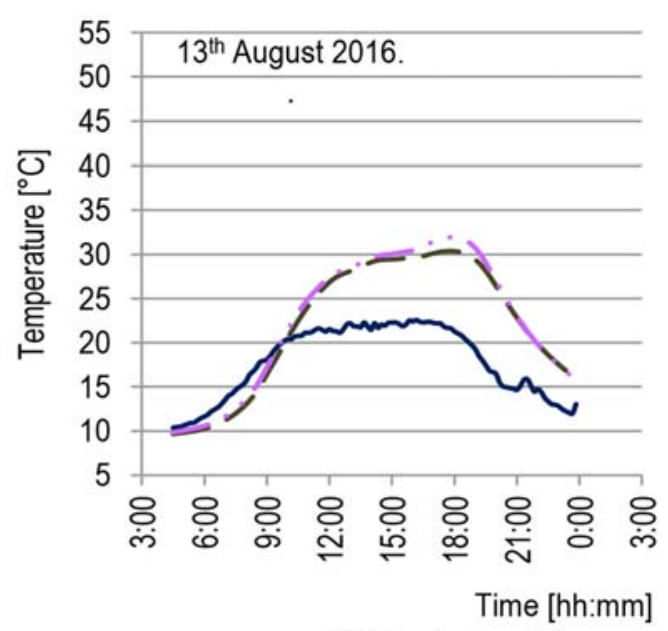

d) West orientation

Legend:

- Inner air temperature of the hut with added convex window - shading device in the buffer zone

_ - - Inner air temperature of the traditional hut - the inner shading device indoor the hut

- ... Inner air temperature of the traditional hut - the outside shading device in front of the window Outside air temperature

Fig. 9. Summer measurements of the experimental huts (time: Central European Time, CET) 
ficult to maintain) on usual windows, not to mention the inner side shadings.

At last we need to mention the urban heat island intensity. In summer afternoons because of the external air curtain mode the solar gain causes upward flow, which increases the urban wind during the day. The solar energy absorbed by the buildings - absorbed during the day and the heat, and emitted after sunset - is lower. Due to the beneficial effect of the external air curtain mode of the added convex windows the urban heat island intensity decreases: this effect is worthy of further analysis.

\section{Acknowledgements}

The work of Attila Kerekes is supported by the EFOP3.6.1-16-2016-00022 project. The project is co-financed by the European Union and the European Social Fund.

\section{References}

[1] Kerekes A. (2017), Impact of added convex windows on energy need for heating in different climatic zones. In: De- sign to Thrive. Proceedings of PLEA 2017 Conference, eds. Brotas L., Roaf S., Fergus N., PLEA, Edinburgh, pp. 628-634.

[2] Kerekes A. (2017), Ablakcsere vs. előtétablak. Magyar Épületgépészet, LXVI(4), 8-12.

[3] Kerekes A., Zöld A. (2016), Added convex windows for improving the energy balance. Applied Mechanics and Materials, 824, 339-346.

[4] Kalmár F. (2016), Interrelation between glazing and summer operative temperatures in buildings. International Review of Applied Sciences and Engineering, 7, 51-60.

[5] Kalmár F. (2016), Summer operative temperatures in free running existing buildings with high glazed ratio of the facades. Journal of Building Engineering, 6, 236-242.

[6] Duffie, J. A., Beckman, W. A. (2013), Solar Engineering of Thermal Processes. 4th Edition, John Wiley \& Sons, Inc., Hoboken, New Jersey, pp. 963.

[7] Spencer, J. W. (1971), Fourier series representation of the position of the sun. Search, 2(5), 172.

[8] Iqbal, M. (1983), An Introduction to Solar Radiation. Academic, Toronto

[9] Erbs D. G., et al. (1982), Estimation of the diffuse radiation fraction for hourly, daily and monthly-average global radiation. Solar Energy, 28(4), 293-302. 